such cells do not release progeny tumour viruses. Before large amounts of time, effort and money are spent infecting human tumour cells with VSV it would be advisable to prove that, for example, VSV pseudotypes are produced when Cofal positive chick cells, which contain an endogenous avian leukosis virus genome, which contain both group and subgroup (envelope) leukosis virus antigens but which do not release leukosis virus particles, are infected with VSV. Such non-producer cells are a more appropriate model of human tumour cells, which also do not liberate detectable amounts of C-type viruses, than cells supporting the complete replication of avian myeloblastosis virus or murine leukaemia virus.-From our Cell Biology Correspondent.

\section{RECEPTORS}

\section{Applied Affinity}

from our Molecular Biology Correspondent A. E. Housman once began a book review: "There is much in this book that is new and much that is true ; unfortunately nothing that is new is true, and nothing that is true is new". The burden of a clutch of papers just out -simultaneously and with evidence of haste, for they are all in quick-fire journals - on the isolation of the acetylcholine receptor by affinity chromatography, falls into the true though not new category, which especially in a tricky and competitive field is the better alternative. The wide measure of agreement between the results of the three independent groups, and those of other workers, earlier reported (and touched on a few weeks back in this column), is distinctly reassuring.

The acetylcholine receptor has been extracted from the electric eel by one group, and from the electric fish, Torpedo, by two others. Olsen, Meunier and Changeux (FEBS Lett., 28, 96; 1972) have made an affinity column by coupling an acetylcholine analogue to 'Sepharose' beads, derivatized to carry reactive thiol groups. The tissue extract in non-ionic detergent is applied to a column of this material, and the bulk of the total protein washes through. The receptor activity and acetylcholinesterase are both retained, however, and the former can be collected by elution with a competing ligand, flaxedil. Concentrated salt serves to dissociate the adsorbed acetylcholinesterase from the matrix. The receptor can be assayed by its capacity to bind the radioactively labelled venom protein, $\alpha$-bungarotoxin, the complex being retained on membrane filters. The up- shot is that half the total receptor sites found in the tissue extract are recovered from the affinity column. The resulting purification factor is about 150 , which is not, however, sufficient to generate anything like a homogeneous preparation, as judged by electrophoretic analysis in SDS-gels.

A rather higher purification is reported with the Torpedo receptor, caught on a column of cobrotoxin coupled to 'Sepharose' (Karlsson, Heilbronn and Widlund, ibid., 107). After extraneous proteins have been eluted, the receptor is dissociated from the toxin by a gradient of carbamylcholine. The striking feature of the elution curve is that there are two peaks, both of which have receptor activity, binding tubocurarine with high efficiency. There is no acetylcholinesterase activity to speak of. The second component has the greater affinity for tubocurarine, but neither is altogether pure, for there is evidence of a background of other proteinaceous material.

The combining weights with toxin correspond to one site per 300,000 and $140,000 \mathrm{~g}$ respectively of the two components. Gel filtration of these components, carrying labelled venom, provides an estimate of their molecular weights (assuming a more or less symmetrical molecule), and both come out at about 140,000 . This is most probably then the molecular weight of the receptor, and it agrees with earlier estimates. The first peak from the affinity column is assumed to contain an inactive protein species. Karlsson et al. claim a purification of a thousand-fold. Half of the toxin-bound material, however, fails to elute in the carbamylcholine medium, and remains stuck to the column. Karlsson et al. offer the attractive, if insecurely founded, suggestion that the receptor might have two subunits, which can be of either of two kinds, only one of which is capable of binding such analogues as carbamylcholine. A not unimportant technical point seems to be that the preparation fails if done in the cold, because, Karlsson et al. suggest, the association with the column-bound toxin is then weak.

The third, and to an extent complementary, description of an affinity-based isolation of receptor, again from Torpedo, comes from Schmidt and Raftery (Biochem. Biophys. Res. Commun., 49, 572 ; 1972). The approach is that of Olsen et al., in that the column-bound ligand is not a protein toxin, but a cholinergic species. The retention is not strong, however, and a dilute salt solution is enough to desorb the trapped receptor activity. The corresponding purification, in terms of $\alpha$-bungarotoxin

\title{
Synthesis of Mitochondrial RNA Polymerase
}

IN next Wednesday's Nature New Biology (December 13), Barath and Küntzel report the latest instalment of their exemplary investigations of the RNA polymerase in mitochondria of Neurospora crassa. Their experiments lead them to the interesting conclusion that mitochondrial RNA polymerase is not only synthesized by cytoplasmic rather than by mitochondrial ribosomes but also that this protein is specified by a nuclear rather than a mitochondrial gene. As Küntzel and Schäfer reported last year in Nature New Biology, the RNA polymerase in Neurospora crassa mitochondria is resistant to $\alpha$ amanitin but sensitive to rifampicin and is composed of polypeptide chains with a molecular weight of 64,000 .

To decide whether this polymerase is specified by mitochondrial DNA or by cell DNA, Barath and Küntzel measured the synthesis of mitochondrial RNA polymerase in cells exposed to ethidium bromide or chloramphenicol, drugs which selectively inhibit transcription and/or translation of mitochondrial DNA. They found that in the presence of these drugs, mitochondrial RNA polymerase is synthesized in large amounts, and accumulates in the cytcplasm of the cells. By contrast, in the presence of ethidium bromide, biosynthesis of cytochrome is inhibited.

These facts indicate that whereas the cytochromes are products of transcription and translation of mitochondrial DNA, mitochondrial RNA polymerase is synthesized in the cell cytoplasm and is coded by a nuclear gene. Apparently inhibition of mitochondrial transcription and translation induces excessive production of mitochondrial RNA polymerase so that cells which have been exposed to ethidium bromide for about $20 \mathrm{~h}$ contain in their cytoplasm crystals of protein which have the same molecular weight and the same electrophonetic mobility as RNA polymerase isolated from mitochondria.

This induction of excessive production of mitochondrial RNA polymerase by ethidium bromide leads Barath and Küntzel to propose a model in which a repressor-like mitochondrial gene product binds to nuclear DNA and restricts synthesis of mitochondrial RNA polymerase. They suggest that when the synthesis of this mitochondrial gene product-the repressor-is blocked, the nuclear genes coding mitochondrial RNA polymerase are continuously expressed so that a large excess of mitochondrial RNA polymerase accumulates in the cell cytoplasm. 